\title{
A Self-contained and Self-explanatory DNA Storage System
}

\section{Min Li}

Shenzhen Institutes of Advanced Technology

Junbiao Dai

Shenzhen Institutes of Advanced Technology

Qingshan Jiang

Shenzhen Institutes of Advanced Technology

Yang Wang ( $\square$ yang.wang1@siat.ac.cn )

Shenzhen Institutes of Advanced Technology

\section{Research Article}

Keywords: DNA storage, self-explanatory method, data loss, random read operations

Posted Date: February 2nd, 2021

DOl: https://doi.org/10.21203/rs.3.rs-154242/v1

License: (c) (1) This work is licensed under a Creative Commons Attribution 4.0 International License. Read Full License 


\title{
A self-contained and self-explanatory DNA storage system
}

\author{
Min $\mathrm{Li}^{1,2}$, Junbiao Dai ${ }^{1}$, Qingshan Jiang ${ }^{1}$, and Yang Wang ${ }^{1,2, *}$ \\ ${ }^{1}$ Shenzhen Institutes of Advanced Technology, Chinese Academy of Sciences \\ ${ }^{2}$ University of Chinese Academy of Sciences \\ *Corresponding author: yang.wang1@siat.ac.cn
}

\begin{abstract}
Current research on DNA storage usually focuses on the improvement of storage density with reduced gene synthesis cost by developing effective encoding and decoding schemes while lacking the consideration on the uncertainty in ultra long-term data storage and retention. Consequently, the current DNA storage systems are often not self-containment, implying that they have to resort to external tools for the restoration of the stored gene data. This may result in high risks in data loss since the required tools might not be available due to the high uncertainty in far future. To address this issue, we propose in this paper a self-contained DNA storage system that can make self-explanatory to its stored data without relying on any external tools. To this end, we design a specific DNA file format whereby a separate storage scheme is developed to reduce the data redundancy while an effective indexing is designed for random read operations to the stored data file. We verified through experimental data that the proposed self-contained and self-explanatory method can not only get rid of the reliance on external tools for data restoration but also minimize the data redundancy brought about when the amount of data to be stored reaches a certain scale.
\end{abstract}

\section{Introduction}

With the prevalence of big data-based applications, the massive quantities of data created across the globe each year are increasing in an exponential fashion ${ }^{1}$, and it is expected that the global data storage demands will rapidly rise to $175 \mathrm{ZB}{ }^{2}$ by 2025, with 2.5 extra bytes per day, far exceeding the world's capacities that traditional storage technologies can afford ${ }^{3}$.

On the other hand, given the uniqueness of gathered big data, it is often desired to achieve them in external storage devices for value extending over a long period of time. To address these challenges, synthetic DNA is emerging as a novel storehouse ${ }^{4-8}$ of information as it is the only potential to be orders of maginitidue denser ${ }^{9}$ than contemporary cutting-edge techniques but also extremely stable to retain the information for hundreds of thousands of years ${ }^{10}$, compared to hard drives which might last only 50 years $^{11,12}$.

Although it is a distinguished medium to store enormous data over millennia by virtue of its inherent high density ${ }^{12-15}$ and durable preservation ${ }^{16,17}$, synthetic DNA is still hardly practical as of today to store more than several hundred megabytes because of the high cost of DNA synthesis ${ }^{18}$ within this field. Consequently, most current research efforts are inspired to explore effective encoding and decoding schemes to facilitate the improvement of DNA storage density with reduced gene synthesis cost as a goal ${ }^{15,19-25}$ For example, a common practical scheme is to compress a digital file before it can be translated into DAN sequence with added error correction ${ }^{15,19,20,26,27}$ as the payload sent to the synthesis module so that the sequence payload can be instantiated into physical DNA molecules.

Although they are beneficial to storage density improvement while reducing the synthesis cost, these schemes might result in high risks in data loss for ultra long-term retention since the DNA storage systems are not self-contained anymore in the sense that the restoration of the stored gene data has to rely on external tools, say decompression program in our example, which might not be available due to the high uncertainty in the far future. As a result, without solving this external reliance issue, it is unlikely for DNA storage to become a viable option for storing and retaining data in ultra-long terms.

In this work, to address the external reliance issue while improving the storage density, we propose a self-contained DNA storage system that can make self-explanatory to its stored data without relying on any external tools. To this end, we allow the external tool, say the corresponding decompression program, to be encoded with the compressed file together into a unified DNA sequence payload. However, unlike the one-to-one mapping between the compressed file and its corresponding decompression program in traditional cases, we deliberately make it possible to share a single decompression program among a set of compressed files for minimizing the information redundancy. Although this strategy is not difficult to implement in traditional storage, it is hard to implement for the DNA storage given its random read hurdles and high sequencing cost in the data restoration. To address these issues and achieve our goal, we design a specific DNA file format whereby a non-continuous storage scheme is developed to reduce the data redundancy on the one hand, and combine with traditional storage media on the 
other hand to obtain effective indexing for random read operations to the stored data file while minimizing the cost with an one-off sequencing.

\section{Methods}

In order to have a full play to the advantages that DNA can store data for an ultra-long time, we propose a concept of self-contained and self-explanatory technology for the DNA storage and design a method to implement it.

Since data compression is an important tool in the DNA storage for cost-efficiency, we concentrate in this research on the proposed technology by taking compression and self-extracting as a focus. In fact, the compression tool can also be stored with other data related information, such as encoding parameters, file storage format, etc. We describe our methodology in three steps. We first overview the DNA storage process in Figure 2, and then introduce the detailed information regarding the data self-containment technology. Finally, we describe the data self-explanation technique by defining the format of the DNA file and DNA fragment to support the implementation of the functions presented in Figure 2.

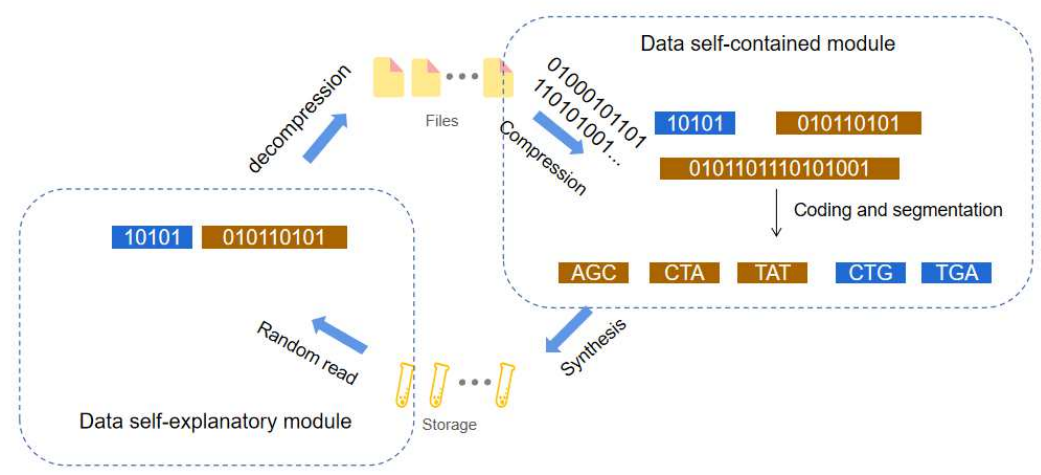

Figure 1 depicts the storage process where the input binary data file is often compressed to minimize the data redundancy while saving the synthesis cost. In order to achieve data selfcontainment, we store both the compressed data and the decompression program as the payloads in the DNA file. The binary data in brown represents the compressed data, and in blue represents the decompression program. Both types

Figure 1. Overview of DNA storage process of data are segmented and uniformly coded as synthetic DNA sequence. Since it is necessary to

distinguish between the data file and the program file in DNA fragments, we deliberately add a $b p$-length flag in the DNA fragments. The data self-explanatory process is reflected in the data restore process and supported by the defined data format.

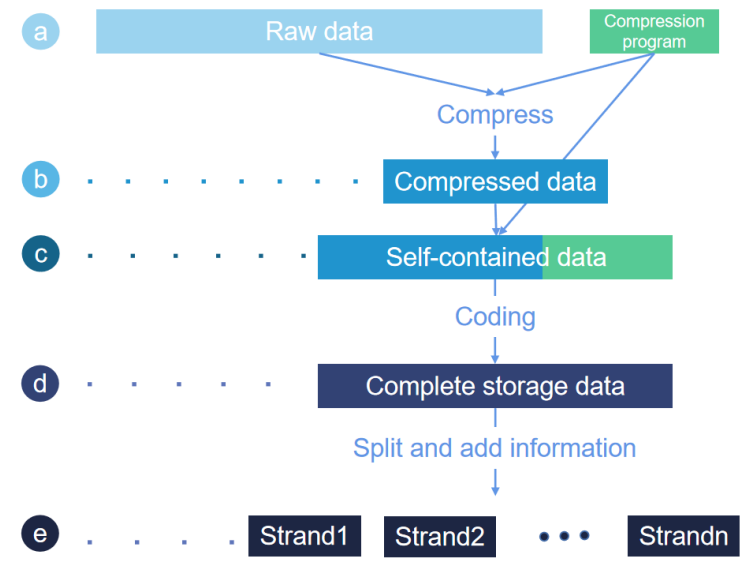

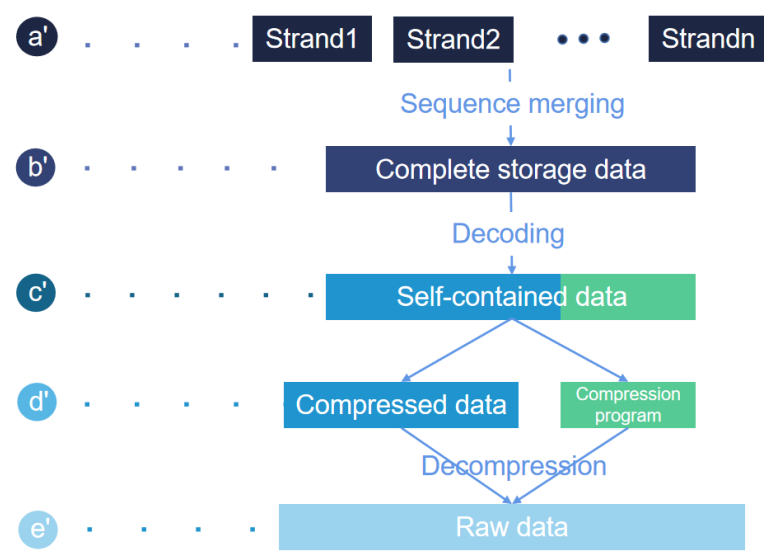

Figure 2. DNA storage process. Figure 2 a shows how the dataflow is changed at each stage of the process. The compressed data (b) and the decompression program constitute the self-contained data (c), which can be simply combined into a single continuous file. However, this change might cause some problems, we will describe them in details later. After encrypting, encoding, and error correcting, we can obtain a long DNA sequence (d), which is then split into a set of short sequences (e), which are finally stored through the artificial synthetic process. In contrast, Figure $2 \mathrm{~b}$ shows how the dataflow change with respect to the reading process. The fragments are first merged into a complete long sequence (b'), which is then decoded to obtain the corresponding self-contained data (c'). The self-contained data is split into compressed data and a compression encoded program (d'), which is used to decompress the compressed data to obtain the original data (e') 


\section{Data Self-Containment}

The existing DNA storage systems are in general not self-contained as they always resort to external tools to backup/restore the data. For example, if the compressed data needs to be restored, the corresponding decompression program should be available. Although the chance of unavailability of the external tool is very small, we may still take the risk to lose the stored data in the case that the required tool is unavailable due to the uncertainties after an ultra-long time period for the DNA storage, say over hundred years. Therefore, we hope that the data and related tools stored in DNA are as complete as possible, allowing the data to be self-contained.

The purpose of our self-containment and self-explanation is to have the DNA file contain more information, not only including the encoded data itself, but also maintaining the index information for the stored data. As shown in Figure 3,4,5, we take the data compression in DNA storage as an example to explain the proposed self-contained and self-explanatory technology. In this example, the decompression program is our tool. As usual, the DNA fragments are stored in pools in disorder, and all the stored files are identified by using primers.

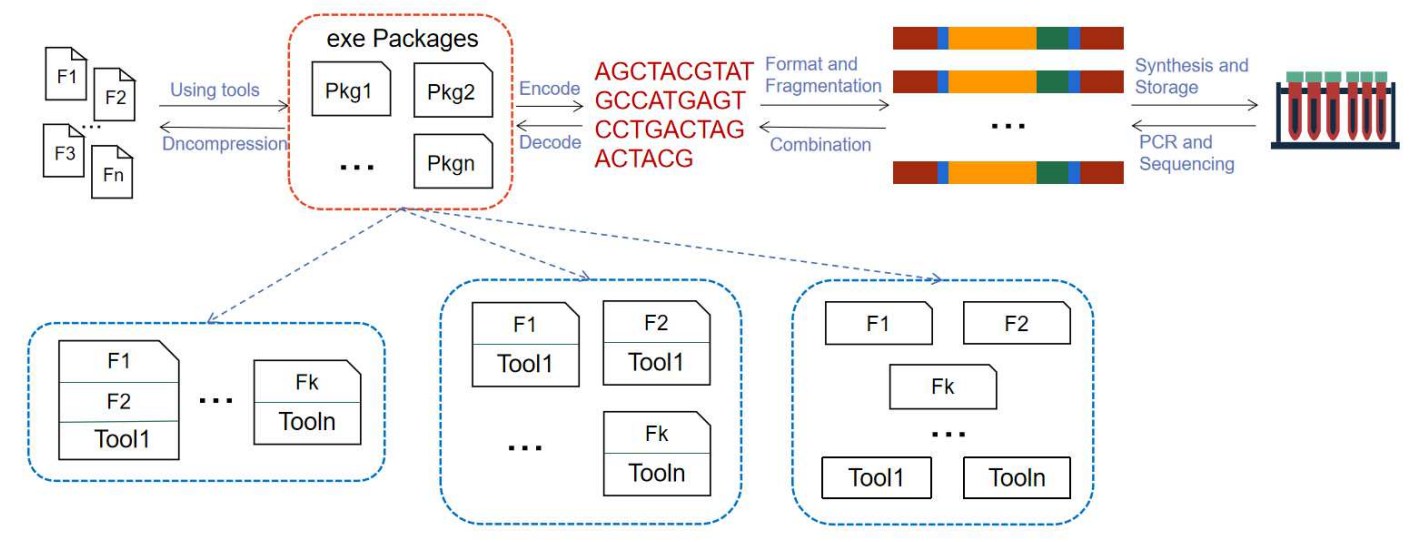

Figure 3. Storage process and three implementation methods: The picture shows the general storage process of DNA files improved with the self-contained method. The red dashed box represents the design of file format while the first blue dashed box below represents intuitive method: combining the data files that adopt the same tool (e.g., using the same compression program) together, for instance, $\mathrm{F} 1$ and $\mathrm{F} 2$ are packaged into a self-extracting program file using compression tool Tool1, and similarly, $\mathrm{Fk}$ is packaged into a self-extracting file using compression tool Tooln. However, this method is not well suitable for the DNA storage. The second blue dashed box indicates that each file is packaged separately which we call this method one-to-One Continuous storage (1-1CS) method, which is also a continuous storage method-the data and tools are stored together. In contrast, the third box represents discontinuous storage method where the tools and data are stored separately(seperate-storage method), which is desired to exploit for minimizing the data redundancy.

\section{Intuitive Method and 1-1CS Method}

The most intuitive method is the one shown in Figure 3's first blue dash box where as with the idea of executable compressed file, the different data files, together with their used program tools, are all packed into a single package, which is formatted as the first blue dotted frame as shown in the figure. However, this method suffers from some drawbacks for the DNA storage because if one file (say, F1) is required, the entire package has to be read and decompressed to obtain the required file. This is difficult to work with the DNA storage as the time and monetary cost of DNA sequencing is much longer and expensive than those of data read from hard drives. As such, we have to enable the DNA storage system to support random read operations for specific compressed files. To address this issue, each file can be compressed independently as shown in the second blue frame where the decompression tool is appended to the compressed data which we called 1-1CS method. Although it can solve the random read issue, this method is still wasteful in space since if multiple files (say, F1 and F2) use the same tool, and the tool has to be saved multiple times, one for each file. Therefore, to minimize the space cost, we reconfigure the executable compressed package by saving the data and the tool program in DNA separately, allowing each of the data files being compressed with the same tool to have a pointer pointing to the same decompression tool file in DNA as shown in Figure 4 or a tool file points to multiple data files related to it as shown in Figure 5. We call these method Many-to-One chain indexing (M-1CI) method and One-to-Many chain indexing (1-MCI) method.

\section{M-1Cl Method}

Compared to the native method, the M-1CI method is fairly effective in minimization of the space cost. However, some practical issues makes it difficult to realize in the PCR and sequencing process in the DNA storage. Since the length of synthetic 
DNA sequence is in general limited to approximately 200 nucleotides, the stored file has to be segmented into a set of short fragments $17,19,20,22$, each being marked with a pair of primers at both ends to index the data stored in between. In this design, the data file needs to hold a pointer (i.e., the tool's primers) pointing to a tool file that can be used to resolve the fragment data. This can be achieved by adding the primers of the tool file to the end of the data file as shown in Figure 4. Although effective in space cost, this design may lead to so-called double sequencing problem.

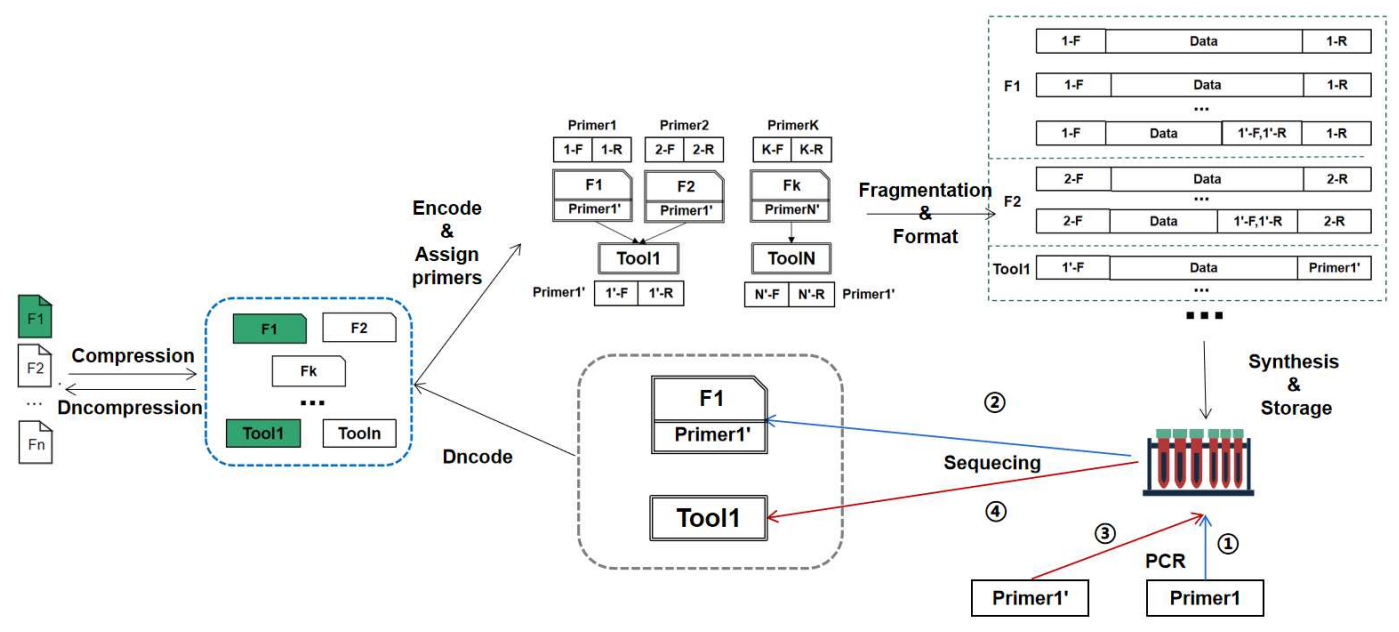

Figure 4. M-1CI Method: As with the previous method, the data is compressed before storage. However, the compressed data files are not continuously stored in a single file as shown in the blue dashed box, instead, they are compressed into separate files. Next, for random read access, we implement a file indexing by adding a file primer to the file (say, Primer 1 to F 1 ), encode the file into a base DNA sequence, then append the tool's primer to the data file (say, Primer $1^{\prime}$ to F1), and finally, perform the sequence segmentation and add tool's primers to each fragment. Suppose in the reverse process if file $\mathrm{F} 1$ is read, the first step (1) is to amplify it by Primer1, then in the second step (2), Primer1' of Tool1, together with F1, can be obtained after a round of sequencing. Then, by using Primer $1^{\prime}$, the tool file is amplified in the third step (3), followed by the fourth step (4) to obtain the Tool1 file by another round of sequencing. After decoding, Tool1 is used to decompress F1 to obtain the original data.

To fully understand the problem, we take an example as shown in Figure 4. In particular, we assign Primer 1 to F1, Primer2 to $F 2$ and Primer $1^{\prime}$ to Tool1, here, PrimerX represents a pair of primers $X-R$ and $X-F$ at the both ends of the sequence, for example, $\operatorname{Primer} 1$ represents a pair of primers $1-F$ and $1-R$ assigned to $F 1$. After the file is encoded into a long DNA sequence, it is then divided into small fragments. At this stage, each pair of primers is actually added to the both ends of the file fragment. The big dashed box in green shows the design of the primer formats of F1, F2, and the tool being used. Each small box represents a collection of file fragments, where the Data field represents the file data (the detailed format will be introduced later).

For example, the first box represents the fragment belonging to $\mathrm{F} 1$. The pointers $1^{\prime}-\mathrm{F}$ and $1^{\prime}-\mathrm{R}$ of $\mathrm{F} 1$ to file $\mathrm{T} \circ \mathrm{O} 11$ are included in the data field of $F 1$, and may be at an end of a fragment. If the data of $F 1$ needs to be read, we need to perform the PCR amplification for the sequence of $\mathrm{F} 1$, according to the known primer Primer 1 . In the second step, the data of F1 is obtained, and then Primer1' of Tool1 is obtained. In the third step, the previous steps are repeated to amplify and sequence to get the Tool1's data according to Primer 1', and finally use Tool1 to process F 1 to restore the original data (e.g., decompress). With this method, one has to restore the data file with 2-round sequencing operations, so-called double sequencing, in which the first round sequencing is to use the selected primers to amplify the specified data file and then sequence it to obtain the primers of the tool file. As the DNA sequencing is time consuming and costly, this double sequencing method is not effective in both time and expenditure.

\section{1-MCI Method:}

In response to the double sequencing problem, we propose a multi-primer method, named One-to-Many Chain Indexing (1-MCI) method that can achieve separate storage for data files and tool files with only one round sequencing to restore the complete data file as shown in Figure 5. To this end, we modify the data format of the DNA fragments so that the tool file can reversely point to the data file. As with the above, we assign Primer 1 to F1, Primer2 to F2, and PrimerK to Fk, 
allow Tool1 file fragments to include Primer1 and Primer2, ToolN to include PrimerK, and enable all tools to share a common known primer Primer $\mathrm{U}^{\prime}$ as the universal primer. The detailed design of the primers after F1, F 2 and ToOl1 segmentation is shown in the large dashed box in Figure 5. Now, the data of F1 and F2 no longer contain the primer of Tool1 as in the 1-MCI method, instead, all the fragments of Tool1 include sub-primers of Primer1 (1-F or 1-R) and Primer2 (2-F or 2-R) and a universal primer Primer $U^{\prime}$, which are used to selectively amplify the file to be read as well as its associated tool. Specifically, with this design, when F1 is read at this time, we only need to amplify the sequence based on the pre-known $\mathrm{F} 1$ 's primers Primer $1(1-\mathrm{F}$ and $1-\mathrm{R})$ and preset the universal primers, and then get $1-\mathrm{F}$ and all the data fragments identified by $1-\mathrm{R}$ belonging to $\mathrm{F} 1$ and all the fragments of Tool1 identified by Primer $\mathrm{U}^{\prime}$ and $1-\mathrm{R}$, thereby directly obtaining the original data with only one round sequencing.

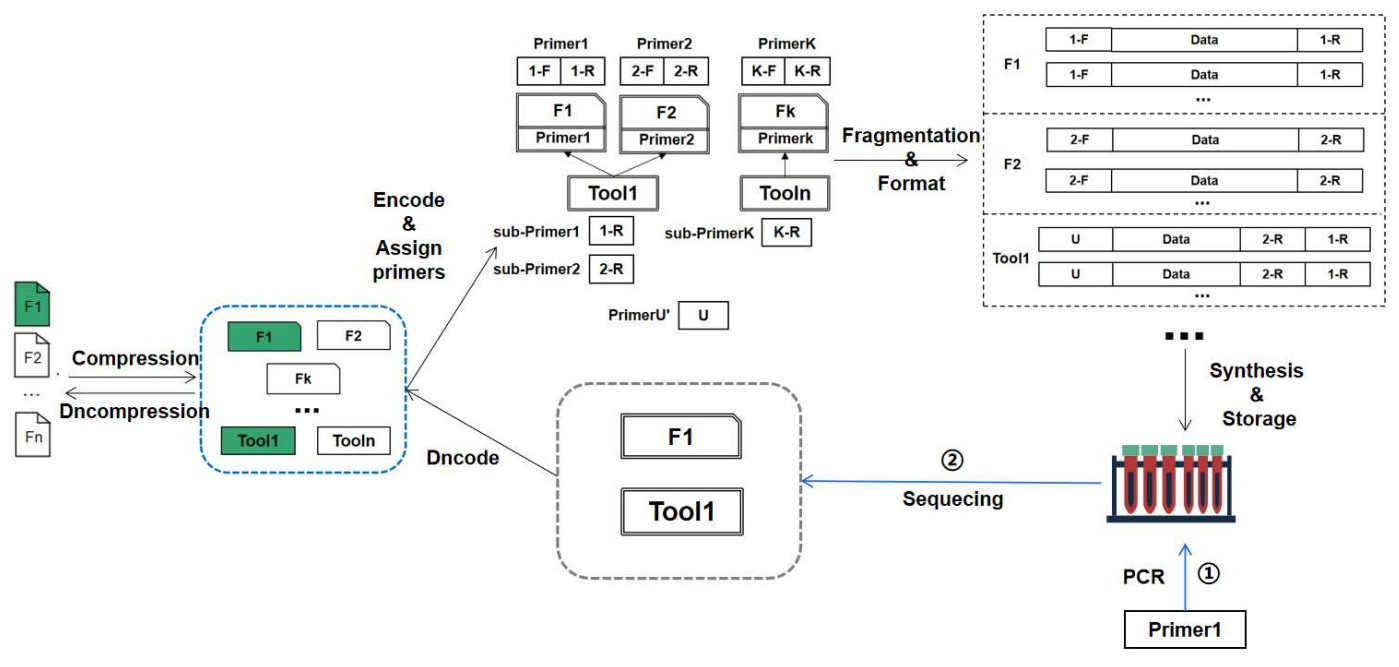

Figure 5. 1-MCI Method: The procedure prior to assigning primers is the same as that of M-1CI. Logically, 1-MCI no longer assigns a specific primer to each tool, rather, all the tools share the same universal primer and include all the primers used in their own data files. The specific implementation is reflected in the sequence after the segmentation is performed, the fragments of all the tool files contain the data file primers and the universal primers of the tools applied to the data files.

Suppose if F1 needs to be read, the first step (1) is to amplify it by Primer 1 , then in the second step (2), sequencing, decoding, and decompressing can be performed once to restore the original file.

The problem with this method is that artificially synthesized DNA fragments have a certain length limitation. As such, if the DNA fragment contains too many program file's primers, the effective data load for the tool file will be reduced. Much worse, if a large number of data files are compressed using the same compression program, the DNA fragments of the tool files may not contain the primers of all the data files. Given this consideration, it is necessary to strike a balance between the self-contained data and the data redundant overload. Overall, even with this problem, the proposed 1-MCI method is still of great significance in practice not only for the correspondence between the tool programs and the data files, but also for other types of file indexes in the DNA storage.

\section{Data Self-Explanation}

The goal of the data self-containment is able to restore the compressed data stored in the DNA storage without relying on external decompression tools. In particular, when reading a data file, the system first finds the file and the primer sequence corresponding to its decompression tools, and then obtains the data and the tool files at the same time through the PCR sequencing technology. After decoding, the decompression tool can automatically restore the data file to its original form to realize the self-explanatory function of the data. Clearly, to achieve this goal, one has to embed many different kinds of information into the DNA file in such a way that the self-contained data is also sufficient to self-explanation, which requires a well defined file format.

\section{Data File Format for Self-Explanation}

The definition of the data format is to support multiple implementations of data self-containment with the ability of selfinterpretation. The data format should include two levels of meta-data information. The first level of the meta-data describes a 
format for the binary compressed file in the data pre-processing step while the second level of the meta-data defines the format for those small fragments after the file is encoded into a DNA sequence.

DNA File Format: The last steps in Figure 4 and Figure 5 are performed to select the specified data files and and their associated tool files. If there are multiple files selected to read at same time, a certain data format is required to support the realization of data self-extraction for these files without compromising others. To this end, we define the format of the data file as shown in Table 1.

\begin{tabular}{|l|l|l|l|}
\hline Field name & Address offset & Size & Description \\
\hline \hline FT & 0 & 1B & File type indicator \\
\hline FID & 1 & 4B & Unique identifier of the file \\
\hline SM & 5 & 1B & Storage method \\
\hline DL or TFID & 6 & 4B & Length of processed data or FID of tool's file \\
\hline D & 10 & Variable length & Length of data \\
\hline TD & Variable length & Variable length & Data of tool \\
\hline
\end{tabular}

Table 1. Data file format. FT means file type, which is either data file or tool file. F ID uniquely identifies a file with an 1-to-1 map to its file name. SM represents three kinds of storage methods-unprocessed file (OF), continuously processed file $(\mathrm{CPF})$, and non-continuously processed file ( $\mathrm{SPF})$. If a file is $\mathrm{OF}$, field $\mathrm{D}$ stores its data, otherwise, if it is $\mathrm{CPF}$, then the next field is DL, specifying the length of the processed data, if it is SPF, the next field is TFID, which means the FID of the used tool file. The last field TD is variable length, if it is CPF, TD means tool data, else the last field length is set to 0 .

In our design, tool file and data file are stored in separate storage. The format of tool file is shown in Table 2.

\begin{tabular}{|l|l|l|l|}
\hline Field name & Address offset & Size & Description \\
\hline \hline FT & 0 & 1B & File type indicator \\
\hline FID & 1 & 4B & Unique file identifier \\
\hline TD & 5 & Variable length & tool's data \\
\hline
\end{tabular}

Table 2. Tool file format. As with the data file, the first field FT specifies file type, the second field F ID is the identifier of the file, and the third field TD is a variable-length field, which stores the data of the tool.

We realize the data self-explanation mainly through the definition of the file format. The logic of file writing is relatively simple, which is simply to write the file in sequence, according to the field orders defined by the file format. The reading process is performed after the DNA data is decoded into binary data. First, the data file and the tool file are separated according to the first field FT, and the data file is put in the datafiles array, the tool file in the toolfiles array. Then, Algorithm 1 for file reading is performed, which first finds the needed file according to FID in the datafiles array ( $\mathrm{Line} 4-5$ ), then determines the storage method of the file according to SM, if SM is OF, the next field is the data field, and the subsequent field is data D, which can be read directly and returned ( $L$ ine 6-7), otherwise if SM is CPF, the data field D could be obtained based on the data length DL of the next field, and the subsequent data is the tool field TD, TD can be used to restore the processed data to the original data and return (Line 8-12), otherwise if SM is SF, the FID of the tool file can be obtained according to field TF ID, then the toolfiles array can be traversed to find TD, whereby the processed data can be restored to the original data ( Line 14-20).

DNA Fragment Format: Due to the limitation of the length of the artificially synthesized DNA sequence, it is necessary to divide the encoded long DNA sequence into small fragments for synthesis. This small DNA fragment consists of multiple fields, as shown in Figure 6.

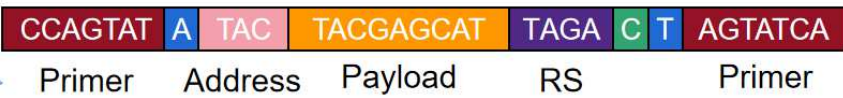

Figure 6. DNA fragment format. The head and tail are primer sequences used to amplify specific file sequences with the PCR technology. The $\mathrm{A}$ and $\mathrm{T}$ at the ends indicate the direction of the sequence, and Addres ${ }^{17}$ gives the offset of the data in the file. The middle Payload field is the data payload, and the RS field in purple is the error correction code. Since PCR can amplify the DNA fragments of the data file and the tool file at the same time, the sequencing result may contain both two files, so field $\mathrm{C}$ in green indicates whether the fragment belongs to the data file or the tool file. 


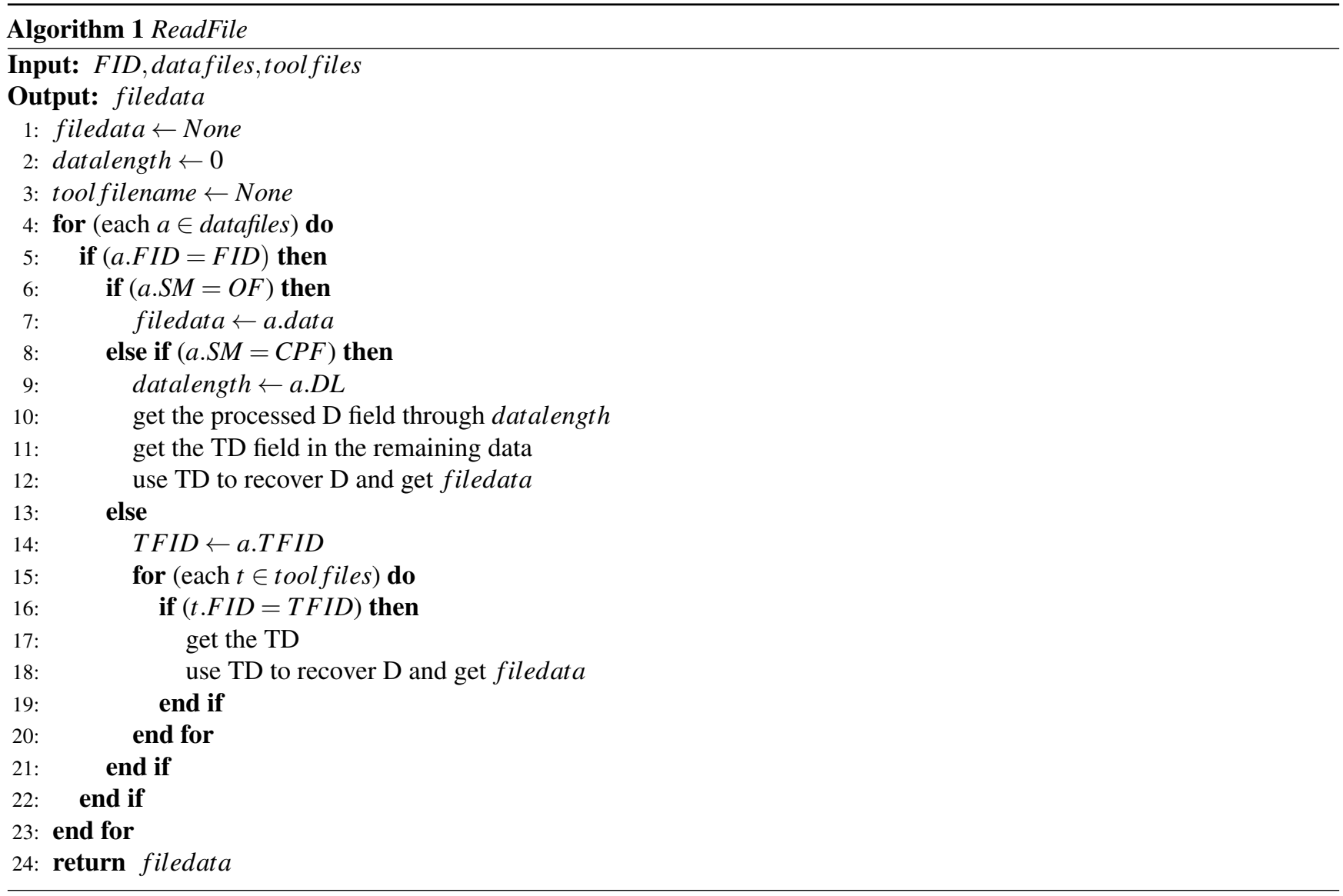

\section{Results}

This section describes the data redundancy brought by the proposed and compared methods and the relevant data volumes of the three data self-containment methods at each stage. The used symbols are listed in Table 3.

\begin{tabular}{|l|l|}
\hline Symbol & Description \\
\hline \hline$r_{c}$ & Compression ratio \\
\hline$S_{o}$ & Size of original data before compressed (bit) \\
\hline$S_{c}$ & Size of compressed data (bit) \\
\hline$e$ & Compression efficiency \\
\hline$e_{o}$ & Compression efficiency of non-self-contained data \\
\hline$e_{c}$ & Compression efficiency of self-contained data \\
\hline$L_{p}$ & Length of primer (bp) \\
\hline$L_{S}$ & Max length of DNA segment (bp) \\
\hline$D i$ & Data file labeled i \\
\hline$S_{D_{i}}$ & Size of data labeled i (bit) \\
\hline$S_{T}$ & Size of tool file (bit) \\
\hline$S_{h}$ & Size of data file's head (bit) \\
\hline$a$ & bp/bit \\
\hline$S b$ & Size of binary data (bit) \\
\hline$S d$ & Number of storage base (bp) \\
\hline$S Q$ & Sequencing volume (bp) \\
\hline
\end{tabular}

Table 3. Symbol description Explanation of symbols to be used 


\section{Data Redundancy}

The data self-containment inevitably brings some redundancy. However, by exploiting the designed structure for separate storage, we can minimize the redundancy to a great extent. To illustrate it, we choose several often-used compression programs as the tool, including rar,7z and zip and calculate the compression ratios $r_{c}$ between them, which is defined as $r_{c}=\frac{S_{o}}{S_{c}} * 100 \%$, here, $S_{o}$ is the original data size and $S_{c}$ is the data size after compressed.

The data self-explanation can be performed with the stored compression program. The redundancy of rar incurred by the self-containment is about $303.5 \mathrm{~KB}$, and $7 z$ is $201 \mathrm{~KB} .7 z$, with the smaller redundancy and good $r_{c}$ as shown in Figure 7 , is used as a test program for our data self-containment redundancy, and file in text format is selected as the test data.

We define the compression efficiency, denoted by $e$, as follows:

$$
e=1-\frac{r_{c} * S_{0}+S_{r}}{S_{0}+S_{r}}
$$

From this definition, one can see that the higher the value of $e$, the better the compression effects, which means that the negative impact of compression program in terms of redundancy is getting smaller and smaller.

We calculate the compression efficiency when the size of the original data $S_{0}$ gradually increases. The compression results of the text file with a size of $500 M B$ show that $r_{c}$ of $7 z$ is 0.34 , the $S_{r}$ of is $201 \mathrm{~KB}$. From Eq. (1), the data storage method without the self-containment is calculated by $e_{o}=1-0.34=0.66$ while the one with the self-containment by $e_{c}=1-\frac{0.34 S_{0}+201 K}{S_{0}+210 K}$. The results of this calculation are shown in Figure 7c.

a

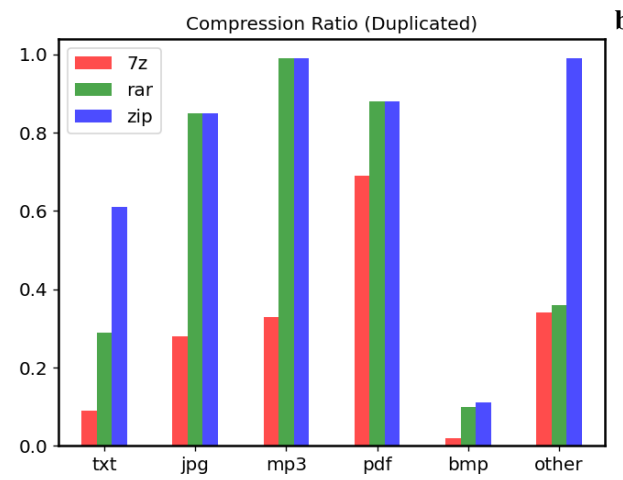

c

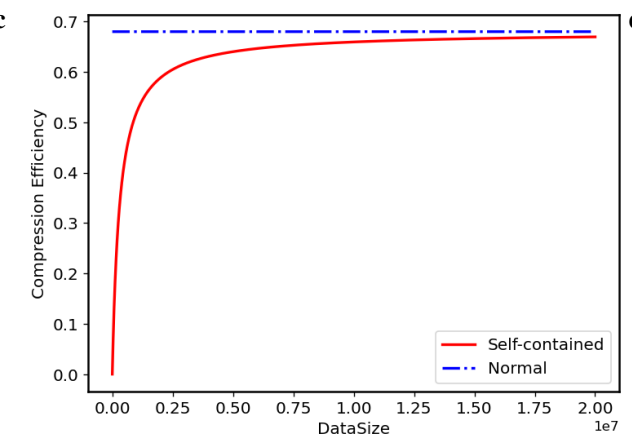

b
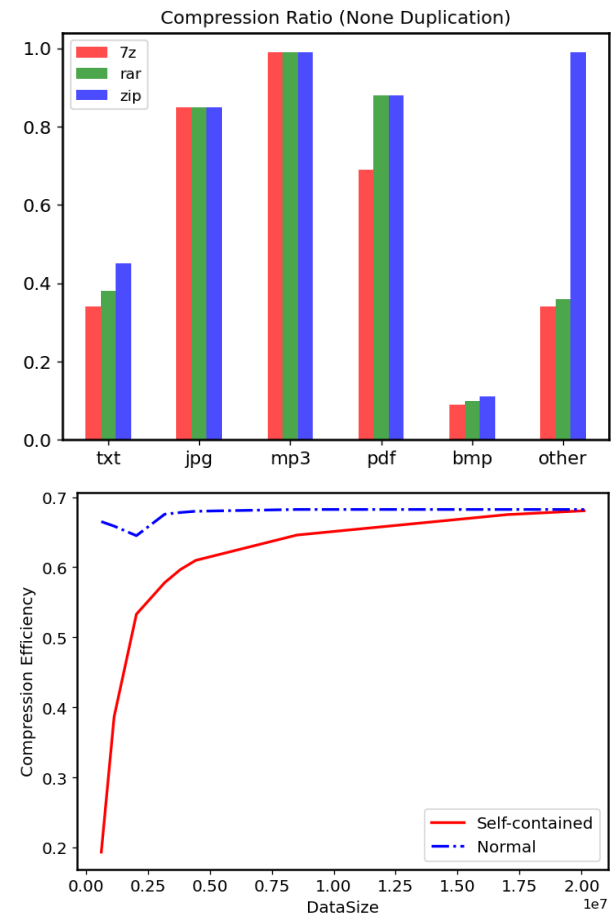

Figure 7. Simulation results. The types of tested data include $t x t, m p 3, j p g, p d f$, and others, such as exe, dll, $h t m l$, etc. Figure 7a shows the $r_{c}$ values of different compression methods. For jpg, $m p 3$, and $p d f$, rar and $z i p$ have the same compression ratio, while the ratio of $7 z$ is better than others. For $t x t$ and $b m p, 7 z$ has obvious advantages. For the $t x t$ and other files, the $z i p$ performs worse. It should be noted that the data we tested contains duplicated file data, so the compression effect of $7 z$ is very good in some cases. We also evaluated $r_{c}$ s of various types using different compression programs without duplicated files as shown in Figure 7b. It can be seen that these three compression programs have the same $r_{c}$ for the jpg and $m p 3$ data. For the $t x t$ and $b m p$ data, the $r_{c}$ s of these three programs are on the rise. $7 z$ has excellent performance on the $p d f$ data, while zip exhibits the worst on others. We tested different types of real data (eg: txt,jpg,exe,mp3) by packing them in a single compressed package as shown in Figure 7d, and the results are roughly the same as the calculated compression efficiency as shown in Figure 7c. It can be seen that when the amount of data reaches a certain scale, the data self-containment has the same effect as those in absence of self-contained methods, and the data redundancy at this time can be almost ignored. 


\section{Data Storage and Sequencing}

We use 1-1CS method and separate-storage method include M-1CI and 1-MCI to achieve the self-contained and self-explanation, since the intuitive method cannot achieve random reading, it is not in the scope of discussion. The separate-storage also has two implementations: M-1CI and 1-MCI, the first method is the most data-saving method, but it requires two PCRs for amplification and sequencing and the second method requires only one amplification and sequencing, but it also brings redundancy to a certain extent. Next, we will discuss the advantages and disadvantages of these two methods under different data relationships.

We first discuss the situation where multiple $n$ data files $\left(D_{i}, i \in 1,2 \ldots n\right)$ are associated with one tool file $T$ in self-contained method. Due to the length limitation of synthetic DNA fragments, the range of $n$ is $0<n<\left\lfloor L_{s} / L_{p}\right\rfloor$. The amount of binary data $S b$ needs to be stored in the 1-1CS method $(s)$, the 1-MCI $(M 1)$ method and the M-1CI (1M) method are respectively:

$$
S b_{s}=\sum_{i=1}^{n} S_{D_{i}}+n *\left(S_{h}+S_{T}\right) \quad S b_{M 1}=\sum_{i=1}^{n} S_{D_{i}}+n *\left(S_{h}+2 L_{p}\right)+S_{T} \quad S b_{1 M}=\sum_{i=1}^{n} S_{D_{i}}+n * S_{h}+S_{T}
$$

In addition to the 1-MCI tool files, the DNA fragments of other files contain a primer at both ends, so the length of the data of the fragment is $L_{s}-2 * L_{p}$, the DNA fragment of the 1-MCI method' tool file contains primers for $n$ data files and a universal primer, so the length is $L_{s}-(n+1) * L_{p}$. The total numbers of bases $S d$ after adding the primers to the segmented fragments produced by the three methods are:

$$
\begin{aligned}
& S d_{s}=S b_{s} * a+\frac{S b_{s} * a}{L_{s}-2 * L_{p}} * 2 L_{p} \\
& S d_{M 1}=S b_{M 1} * a+\frac{S b_{M 1} * a}{L_{s}-2 * L_{p}} * 2 L_{p} \\
& S d_{1 M}=S b_{1 M} * a+\frac{\left(\sum_{i=1}^{n} S_{D_{i}}+n * S_{h}\right) * a}{L_{S}} * 2 L_{P}+\frac{S_{T} * a}{L_{s}-(n+1) * L_{p}} *(n+1) L_{p}
\end{aligned}
$$

Since the M-1CI and 1-MCI methods are superior to the continuous storage method in terms of redundancy, the amount of sequencing is not necessarily the same when reading files at random. Under the assumption that the amount of sequencing of $n$ files is read $n$ times, The number of sequencing in the file units of the three methods is $n, 2 n$ and $n$, respectively, and thus the sequencing amount $S Q$ is:

$$
\begin{aligned}
& S Q_{S}=S b_{s} * a \\
& S Q_{M 1}=\left(\sum_{i=1}^{n} S_{D_{i}}+n *\left(S_{h}+2 L_{p}\right)+n * S_{T}\right) * a
\end{aligned}
$$

Since the fragments of the third tool file contain the primers of all the data files, and the positions of the primers are not the same, the data length between the universal primer (assumed at the left end) and the data file primer (assumed at the right end) is changed, if a file has a high reading frequency, the primer of this file should be placed on the left side. Here we set the same reading frequency for each file. So the sequencing volume of 1-MCI is:

$$
S Q_{1 M}=\left(\sum_{i=1}^{n} S_{D_{i}}+n *\left(S_{h}+2 L_{p}\right)+S_{T}\right) * a+\frac{n-1}{2} * L_{p}
$$

In order to simplify the calculation, we assume that each $D_{i}$ has an equal size $S_{D_{i}}$. The figure shows the quantitative analysis result of the function. We counted the changes in $S_{D_{i}}=10^{3}$ and $10^{5}$. Figure $8 \mathrm{a}$ and Figure $8 \mathrm{~b}$ show how $S d$ is changed as $n$ increases.One can see that with the increase of $n$ in the two figures, the data volume of the 1-1CS method is gradually greater than that of the M-1CI and 1-MCI methods, but in Figure 8b, the data size SD increases $\left(S D=10^{5}>S D=10^{3}\right)$ leads to a reduction in the proportion of $S T$, and ultimately reduces the data volume $S b$ gap between 1-1CS and the two separate storage methods (M-1CI and 1-MCI). Figures 8c and 8d show how the amount of sequencing SQ is changed as $n$ increases-when the 
data size $S_{D_{i}}=10^{3}$ in Figure 8c, the M-1CI needs to be sequenced twice every time, so the amount of sequencing SQ of M-1CI is the same as that of the 1-1CS method, and the 1-MCI method more less than both two methods. When the data size $S D$ increases $\left(S_{D_{i}}=10^{5}\right), \mathrm{M}-1 \mathrm{CI}$ saves the redundant space so that the amount of sequencing is the same as 1-MCI and less than that of 1-1CS.

Generally speaking, compared with the three methods, because 1-1CS needs to store multiple copies of the same tool, it has the disadvantage of large data redundancy, so $\mathrm{M}-1 \mathrm{CI}$ and 1-MCI are better than 1-1CS for $S d$. However, this advantage is more obvious when the data size SD is relatively small and the tool ST is relatively large. If the proportion of SD increases, the advantage will be reduced. In terms of sequencing volume SQ, due to the problem of double sequencing in M-1CI, M-1CI and 1-1CS are the same when the proportion of tools is large. When the proportion of tools is small, the proportion of data is large, M-1CI saves part Redundancy, so it is consistent with 1-MCI. Therefore, 1-1CS is more suitable for the situation where SD accounts for a relatively large proportion, and $\mathrm{M}-1 \mathrm{CI}$ and 1-MCI are more suitable for the situation where SD accounts for a relatively large proportion. Although 1-MCI is optimal in the range of $n$ we are discussing, 1-MCI cannot be used if it exceeds the range of $n$, so $1-\mathrm{MCI}$ is more suitable for situations where the number of files $n$ is small.

a

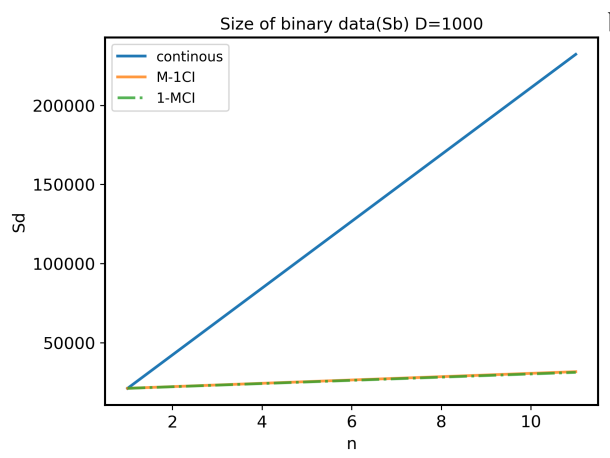

c

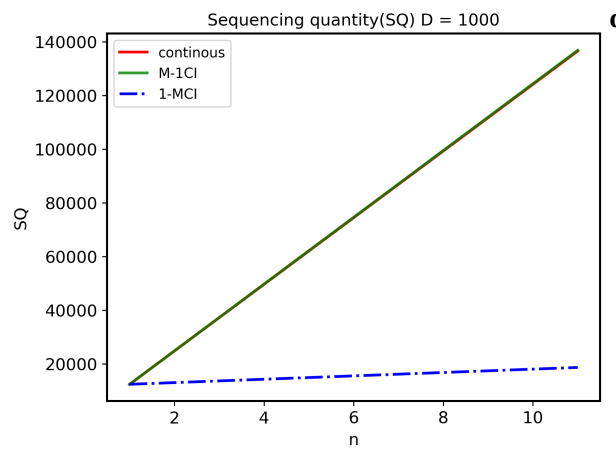

b

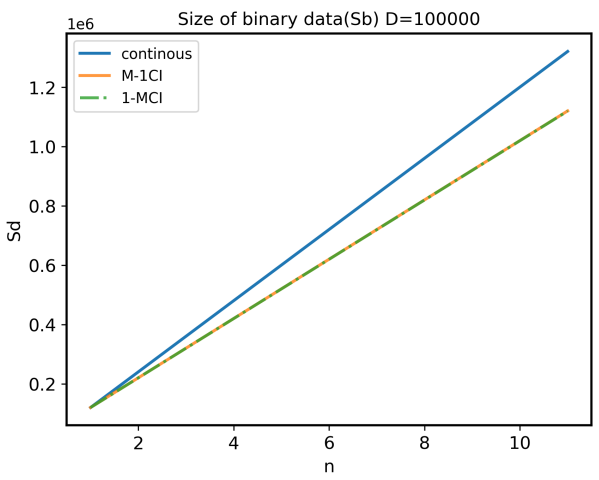

d

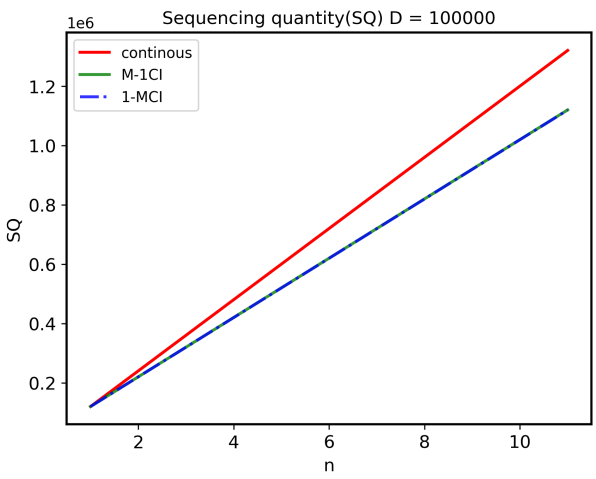

Figure 8. Comparison of data volume and sequencing volume We set the parameters as $S h=10 B, S T=2010 B$, $L p=20 \mathrm{bp}, L s=250 \mathrm{bp}$, and $a=1 / 1.7$ to obtain a comparison chart in the two cases of $D=10 \mathrm{~KB}$ and $D=1 M B$. Figures $8 \mathrm{a}$ and 8bfollow As $n$ increases, the amount of binary data changes in the three methods. Figure $8 \mathrm{c}$ and Figure $8 \mathrm{~d}$ show the changes in the amount of sequencing of the three methods as $n$ increases.

\section{Discussion}

The current DNA storage often resort to external tool to restore the original data from the stored DNA sequence, compromising the data integrity, which is critical to ultra-long-term data archives. To address this issue, we proposed a self-contained and self-explanatory DAN storage in this paper, which not only contains the data to be stored but also includes the tool that is used in pre-processing of the data, say compression program. The proposed system needs to be well designed to address two problems - data redundancy and random read. To this end, we developed the 1-MCI method to minimize the redundant data and defined file formats for both DNA and its fragment files to realize the self-explanation function. We evaluated the proposed system via a prototype implementation and analytical experiments, which show that the redundancy brought about by the self-contained data can be ignored if the data to be stored exceeds a certain scale. Additionally, we also made an in-depth exploration of the file index design with a corresponding file and DNA fragment storage formats.

Although it is proposed to address the data integrity issue for compressed data restoration as a holy grail, our method is 
generic enough to address other issues that may also benefit the DNA storage. For example, embedding the meta-data for the data deduplication ${ }^{28}$ in the DNA storage could dramatically improve the system robustness in uncertain settings while increasing the cost-efficiency, which lay the foundation for the application of the self-contained DNA storage in large-scale data storage.

\section{Acknowledgement}

This work is supported in part by National Key Research and Development Program of China (2020YFA0909100), Shenzhen Key Laboratory of Synthetic Genomics (ZDSYS201802061806209), Guangdong Provincial Key Laboratory of Synthetic Genomics (2019B030301006), and Shenzhen Science and Technology Program (KQTD20180413181837372).

\section{References}

1. Reinsel, D., Gantz, J. \& Rydning, J. Data age 2025: the digitization of the world from edge to core. IDC White Pap. Doc US44413318 1-29 (2018).

2. Reinsel, D., Gantz, J. \& Rydning, J. White paper: The digitization of the world from edge to core. Tech. Rep., Technical Report US44413318, International Data Corporation, Framingham i (2018).

3. Bohannon, J. Dna: the ultimate hard drive. Science (2012).

4. Wiener, N. Machines smarter than men? interview with dr. norbert wiener. noted scientist. US News \& World Rep. 84-86 (1964).

5. Neiman, M. On the molecular memory systems and the directed mutations. Radiotekhnika 6, 1-8 (1965).

6. Clelland, C. T., Risca, V. \& Bancroft, C. Hiding messages in dna microdots. Nature 399, 533-534 (1999).

7. Bancroft, C., Bowler, T., Bloom, B. \& Clelland, C. T. Long-term storage of information in dna. Science 293, 1763-1763 (2001).

8. Yiming, D., Fajia, S., Zhi, P., Qi, O. \& Long, Q. Dna storage: research landscape and future prospects. Natl. Sci. Rev. 6 (2020).

9. Ceze, L., Nivala, J. \& Strauss, K. Molecular digital data storage using dna. Nat. Rev. Genet. 20, 456-466 (2019).

10. Zhi, P. et al. Carbon-based archiving: current progress and future prospects of dna-based data storage. Gigaence 8 (2019).

11. Extance, A. How dna could store all the world's data. Nature 537 (2016).

12. Zhirnov, V., Zadegan, R. M., Sandhu, G. S., Church, G. M. \& Hughes, W. L. Nucleic acid memory. Nat. materials 15, 366-370 (2016).

13. Poltyrev, G. S. Book review. csiszari. and kornerj. "information theory. coding theorems for discrete memoryless systems". Probl. Peredachi Inf., 1982 108\& ndash;111 (1982).

14. Rutten, M. G., Vaandrager, F. W., Elemans, J. A. \& Nolte, R. J. Encoding information into polymers. Nat. Rev. Chem. 2, 365-381 (2018).

15. Organick, L. et al. Random access in large-scale dna data storage. Nat. biotechnology 36, 242 (2018).

16. Allentoft, M. E. et al. The half-life of dna in bone: measuring decay kinetics in 158 dated fossils. Proc. Royal Soc. B: Biol. Sci. 279, 4724-4733 (2012).

17. Grass, R. N., Heckel, R., Puddu, M., Paunescu, D. \& Stark, W. J. Robust chemical preservation of digital information on dna in silica with error-correcting codes. Angewandte Chemie Int. Ed. 54, 2552-2555 (2015).

18. KA, W. National human genome research institute. dna sequencing costs: data from the nhgri genome sequencing program (gsp). http://www.genome.gov/sequencingcosts (2020).

19. Church, G. M., Gao, Y. \& Kosuri, S. Next-generation digital information storage in dna. Science 337, 1628-1628 (2012).

20. Goldman, N. et al. Towards practical, high-capacity, low-maintenance information storage in synthesized dna. Nature 494, 77-80 (2013).

21. Yazdi, S. H. T., Yuan, Y., Ma, J., Zhao, H. \& Milenkovic, O. A rewritable, random-access dna-based storage system. Sci. reports 5, 14138 (2015).

22. Bornholt, J. et al. A dna-based archival storage system. In Proceedings of the Twenty-First International Conference on Architectural Support for Programming Languages and Operating Systems, 637-649 (2016). 
23. Erlich, Y. \& Zielinski, D. Dna fountain enables a robust and efficient storage architecture. Science 355, 950-954 (2017).

24. Blawat, M. et al. Forward error correction for dna data storage. Procedia Comput. ence 80, 1011-1022 (2016).

25. Al-Okaily, A., Almarri, B., Al Yami, S. \& Huang, C.-H. Toward a better compression for dna sequences using huffman encoding. J. Comput. Biol. 24, 280-288 (2017).

26. Reed, I. S. \& Solomon, G. Polynomial codes over certain finite fields. J. Soc. for Ind. \& Appl. Math. 8, 300-304 (1960).

27. Rashtchian, C. et al. Clustering billions of reads for dna data storage. In Advances in Neural Information Processing Systems, 3360-3371 (2017).

28. Xia, W. et al. A comprehensive study of the past, present, and future of data deduplication. Proc. IEEE 104, 1681-1710, DOI: 10.1109/JPROC.2016.2571298 (2016).

\section{Author contributions statement}

M.L. designed and implemented the method and performed the experiments to verify the ideas. Y.W. developed the ideas and conceived the study and assisted M.L. to write the manuscript. Y.W., J.D., and Q.J. directed the experiments and analysed the results. All authors reviewed the manuscript. 


\section{Figures}

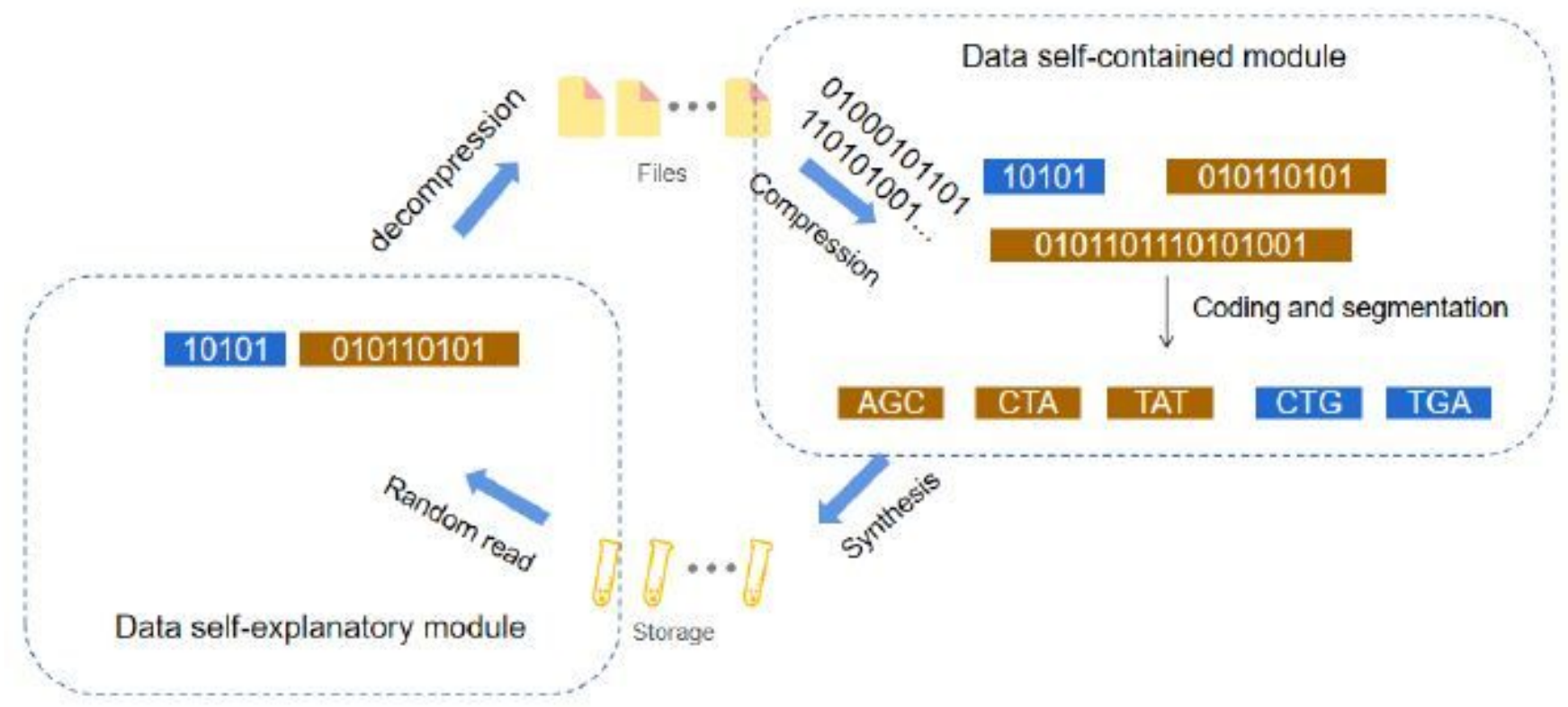

\section{Figure 1}

Overview of DNA storage process

a

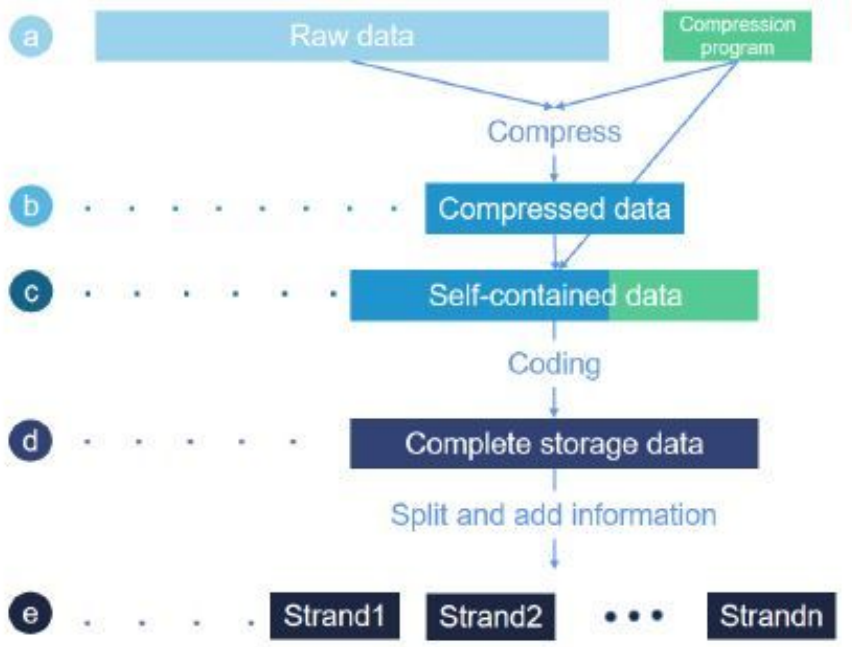

b

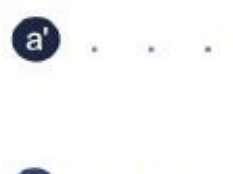

Strand1

Strand2

Strandn

(b)

Sequence merging

Complete storage data

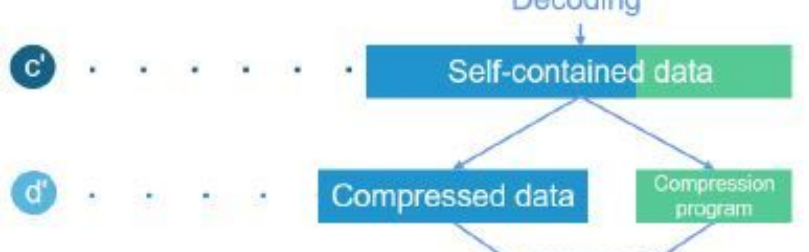

Decompression

\section{Raw data}

\section{Figure 2}

DNA storage process. Figure 2a shows how the dataflow is changed at each stage of the process. The compressed data (b) and the decompression program constitute the self-contained data (c), which can be simply combined into a single continuous file. However, this change might cause some problems, we will describe them in details later. After encrypting, encoding, and error correcting, we can obtain a long DNA sequence (d), which is then split into a set of short sequences (e), which are finally stored through the artificial synthetic process. In contrast, Figure $2 b$ shows how the dataflow change with respect to the 
reading process. The fragments are first merged into a complete long sequence $\left(b^{\prime}\right)$, which is then decoded to obtain the corresponding self-contained data ( $\left.c^{\prime}\right)$. The self-contained data is split into compressed data and a compression encoded program ( $\left.d^{\prime}\right)$, which is used to decompress the compressed data to obtain the original data $\left(e^{\prime}\right)$

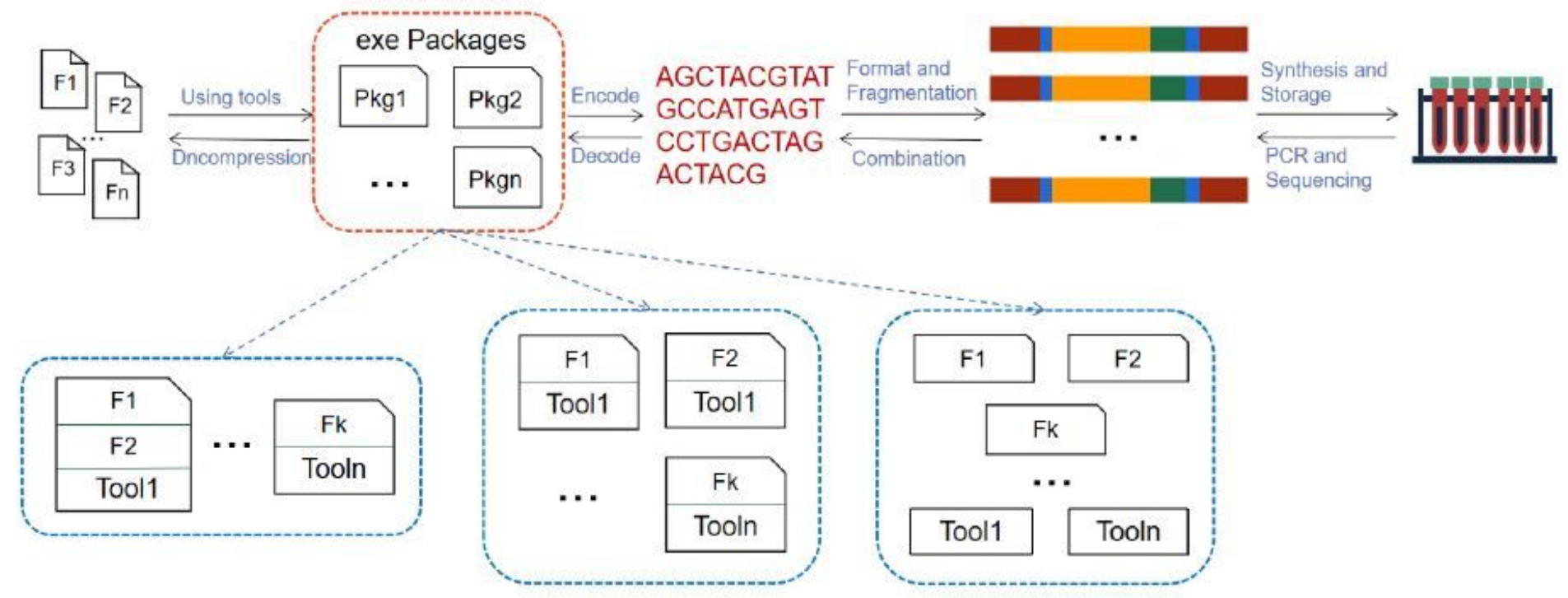

\section{Figure 3}

Storage process and three implementation methods: The picture shows the general storage process of DNA files improved with the self-contained method. The red dashed box represents the design of file format while the first blue dashed box below represents intuitive method: combining the data files that adopt the same tool (e.g., using the same compression program) together, for instance, F1 and F2 are packaged into a self-extracting program file using compression tool Tool1, and similarly, Fk is packaged into a self-extracting file using compression tool Tooln. However, this method is not well suitable for the DNA storage. The second blue dashed box indicates that each file is packaged separately which we call this method one-to-One Continuous storage (1-1CS) method, which is also a continuous storage method -the data and tools are stored together. In contrast, the third box represents discontinuous storage method where the tools and data are stored separately(seperate-storage method), which is desired to exploit for minimizing the data redundancy. 


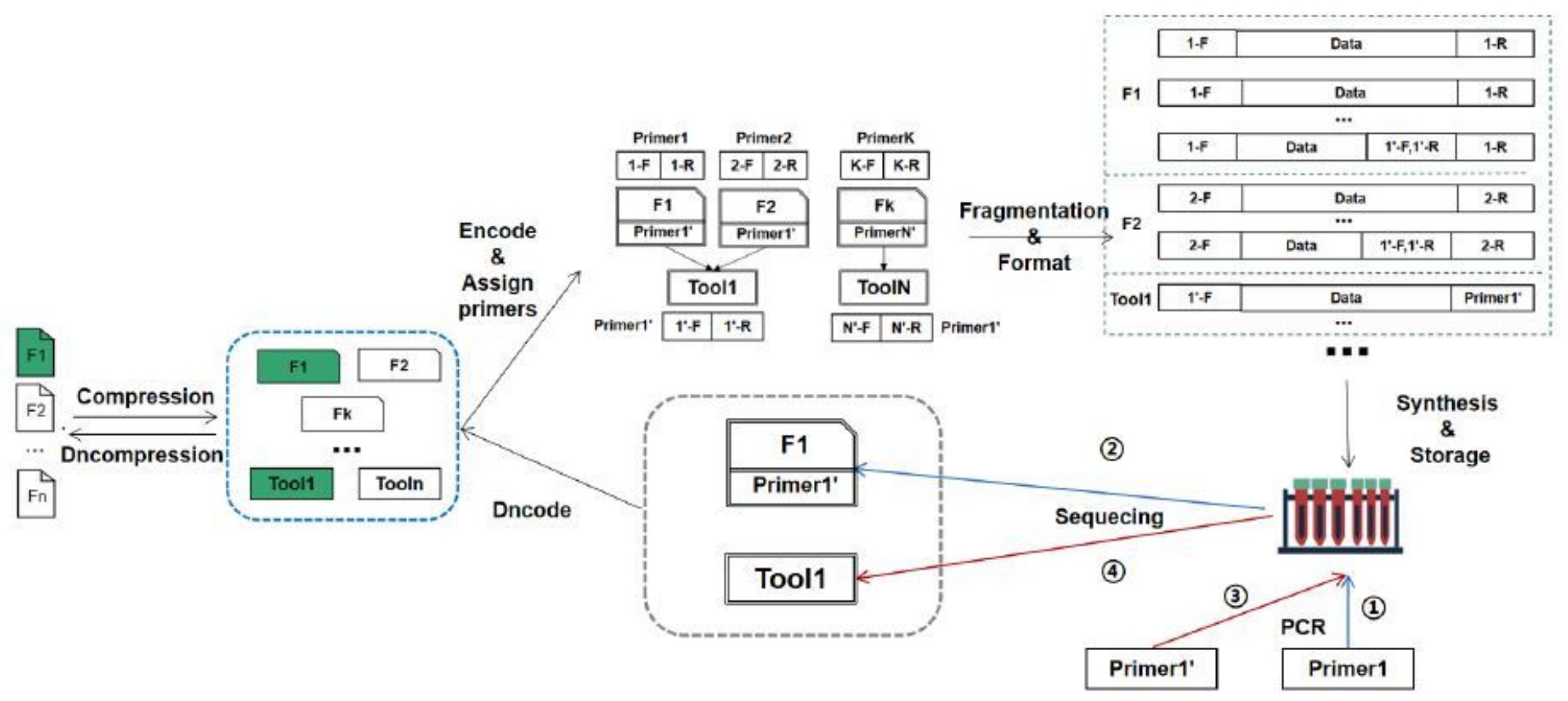

Figure 4

$\mathrm{M}-1 \mathrm{Cl}$ Method: As with the previous method, the data is compressed before storage. However, the compressed data files are not continuously stored in a single file as shown in the blue dashed box, instead, they are compressed into separate files. Next, for random read access, we implement a file indexing by adding a file primer to the file (say, Primer1 to F1), encode the file into a base DNA sequence, then append the tool's primer to the data file (say, Primer1' to F1), and finally, perform the sequence segmentation and add tool's primers to each fragment. Suppose in the reverse process if file F1 is read, the first step 1 is to amplify it by Primer1, then in the second step 2 , Primer1' of Tool1, together with F1, can be obtained after a round of sequencing. Then, by using Primer1', the tool file is amplified in the third step 3 , followed by the fourth step 4 to obtain the Tool 1 file by another round of sequencing. After decoding, Tool1 is used to decompress F1 to obtain the original data. 


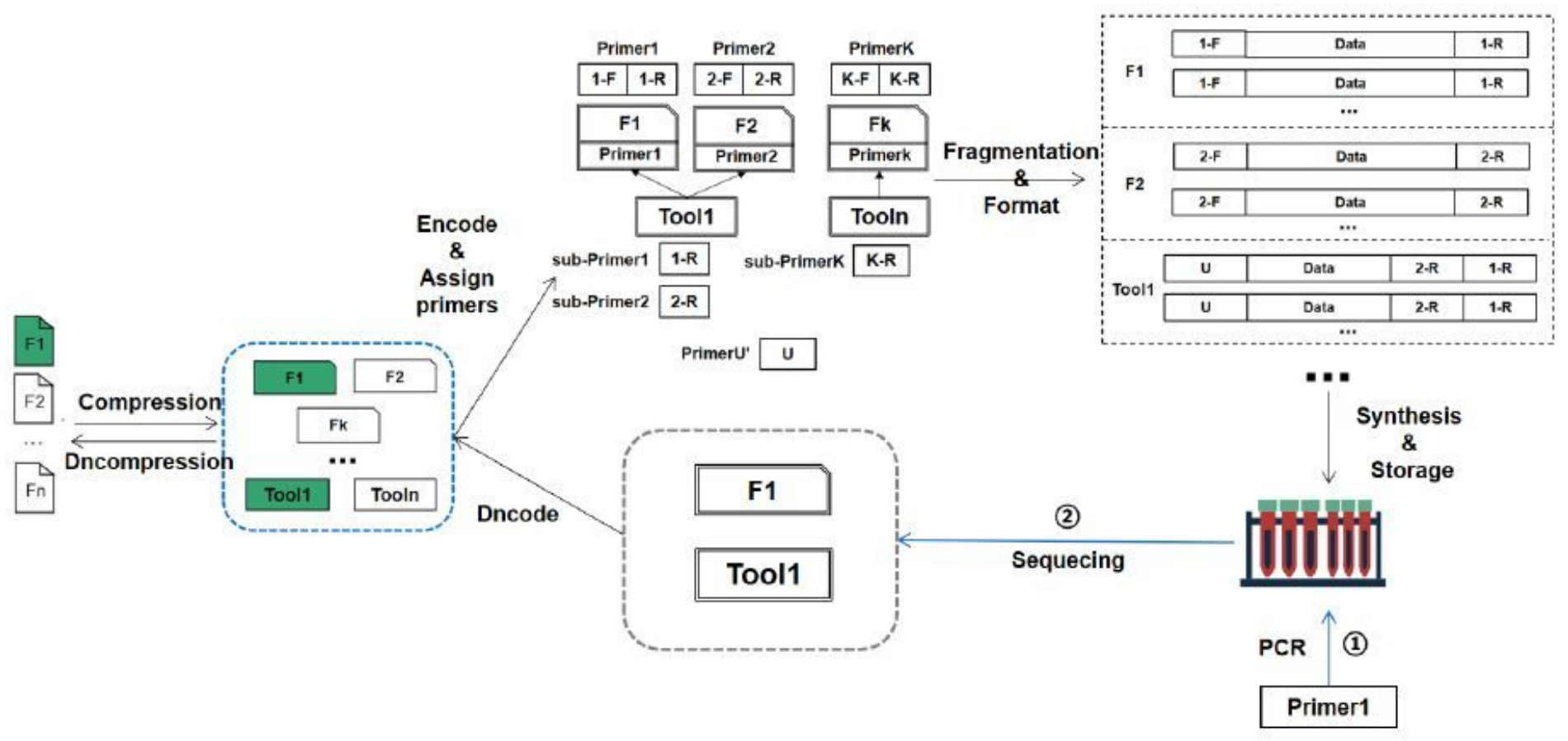

Figure 5

1-MCl Method: The procedure prior to assigning primers is the same as that of $\mathrm{M}-1 \mathrm{Cl}$. Logically, 1-MCI no longer assigns a specific primer to each tool, rather, all the tools share the same universal primer and include all the primers used in their own data files. The specific implementation is reflected in the sequence after the segmentation is performed, the fragments of all the tool files contain the data file primers and the universal primers of the tools applied to the data files. Suppose if F1 needs to be read, the first step 1 is to amplify it by Primer1, then in the second step 2 , sequencing, decoding, and decompressing can be performed once to restore the original file.

\section{\begin{tabular}{l|l|l|l|l|l|l|l|l} 
CCAGTAT & A & TAC & TACGAGCAT & TAGA & C & T & AGTATCA
\end{tabular}}

\section{Primer Address Payload RS Primer}

\section{Figure 6}

DNA fragment format. The head and tail are primer sequences used to amplify specific file sequences with the PCR technology. The A and T at the ends indicate the direction of the sequence, and Address 17 gives the offset of the data in the file. The middle Payload field is the data payload, and the RS field in purple is the error correction code. Since PCR can amplify the DNA fragments of the data file and the tool file at the same time, the sequencing result may contain both two files, so field $\mathrm{C}$ in green indicates whether the fragment belongs to the data file or the tool file. 
a

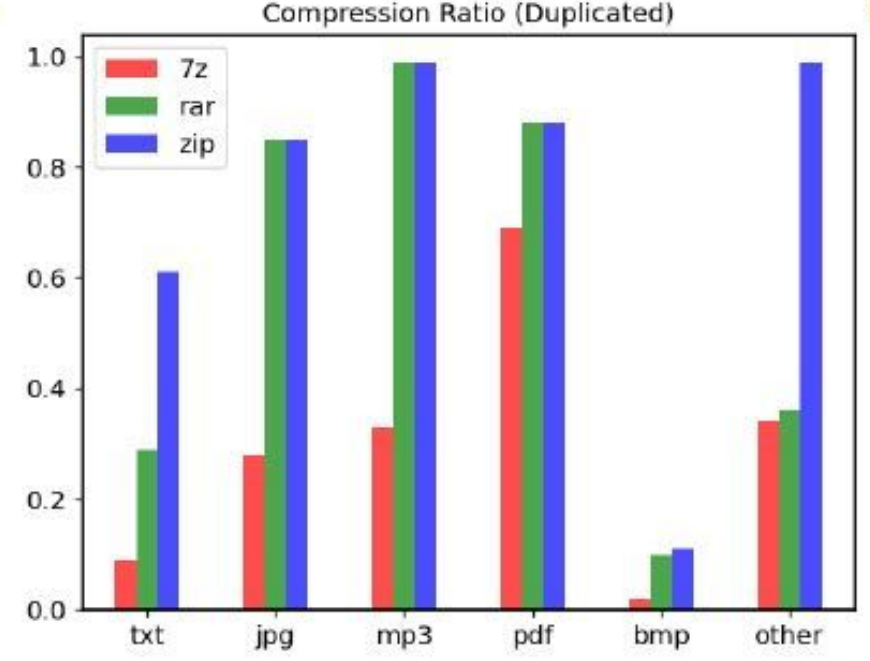

b

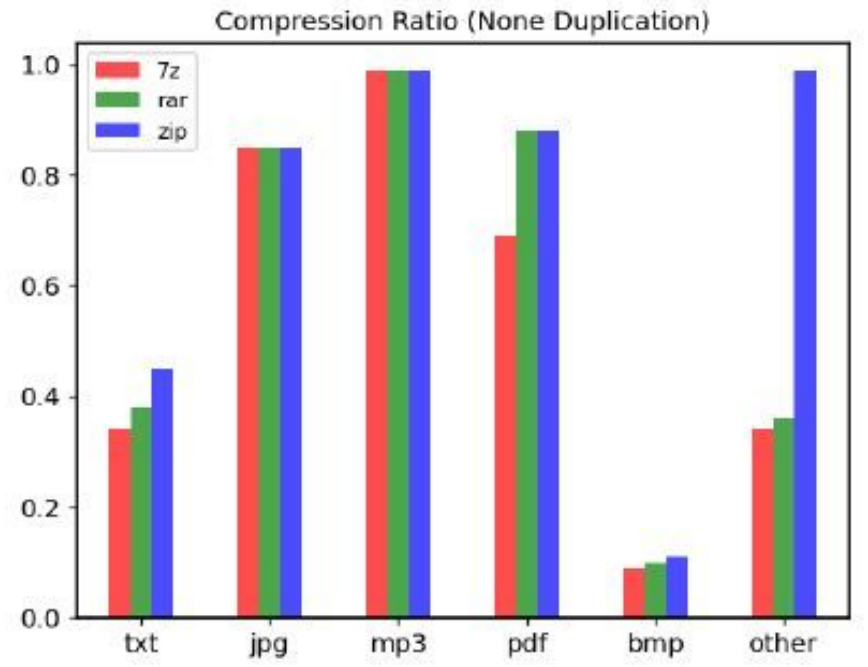

c

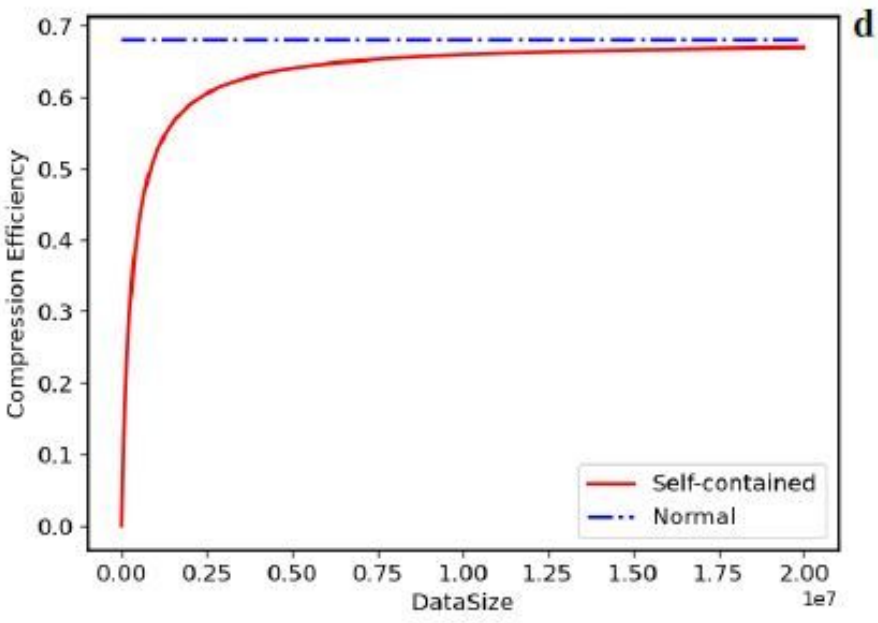

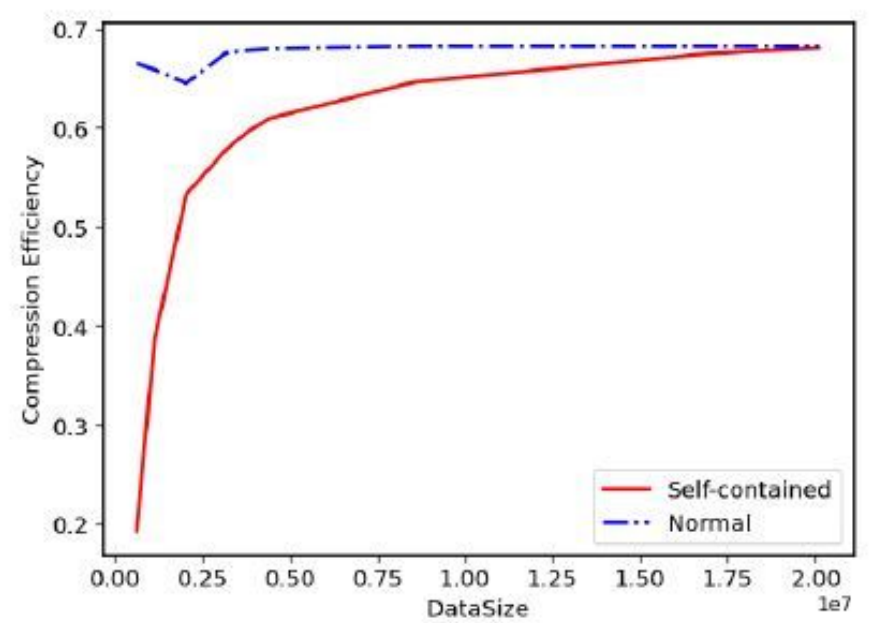

Figure 7

Simulation results. The types of tested data include txt, mp3, jpg, pdf, and others, such as exe, dll, html, etc. Figure 7a shows the rc values of different compression methods. For jpg, mp3, and pdf, rar and zip have the same compression ratio, while the ratio of $7 z$ is better than others. For txt and bmp, $7 z$ has obvious advantages. For the txt and other files, the zip performs worse. It should be noted that the data we tested contains duplicated file data, so the compression effect of $7 z$ is very good in some cases. We also evaluated rcs of various types using different compression programs without duplicated files as shown in Figure 7b. It can be seen that these three compression programs have the same rc for the jpg and $\mathrm{mp} 3$ data. For the txt and bmp data, the rcs of these three programs are on the rise. $7 z$ has excellent performance on the pdf data, while zip exhibits the worst on others. We tested different types of real data (eg: txt,jpg,exe,mp3) by packing them in a single compressed package as shown in Figure 7d, and the results are roughly the same as the calculated compression efficiency as shown in Figure 7c. It can be seen that when the amount of data reaches a certain scale, the data self-containment has the same effect as those in absence of self-contained methods, and the data redundancy at this time can be almost ignored. 
a

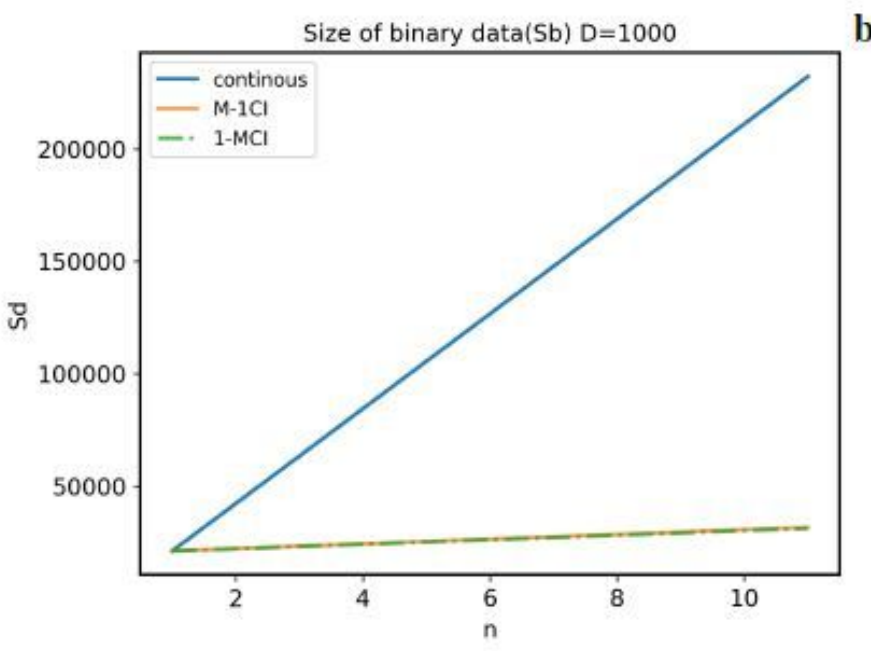

c

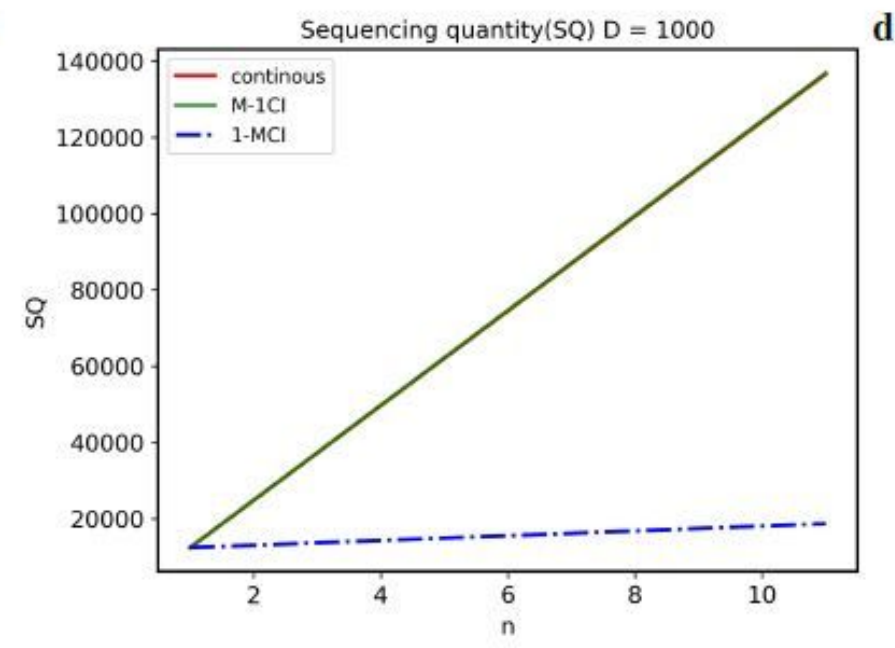

b

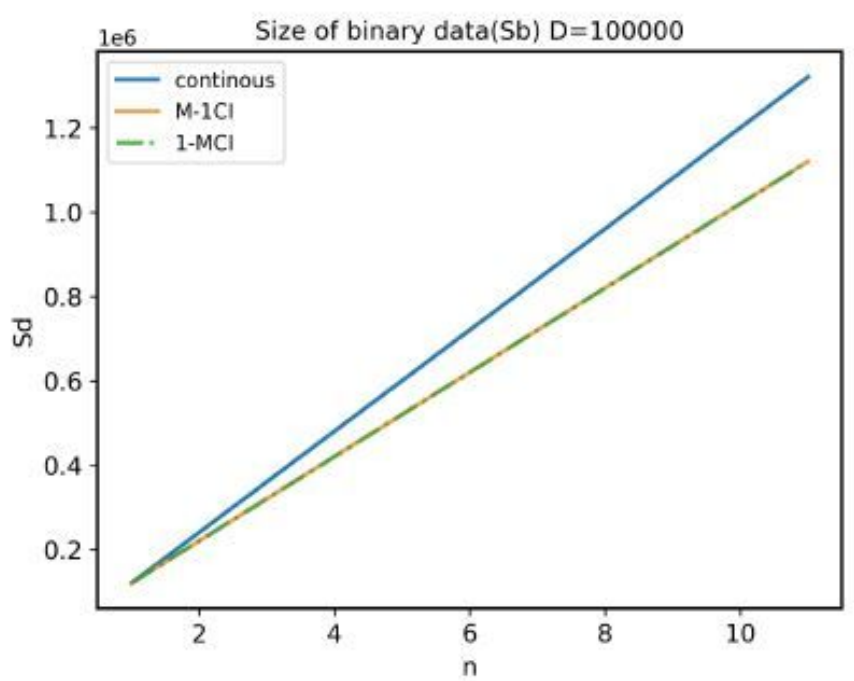

d

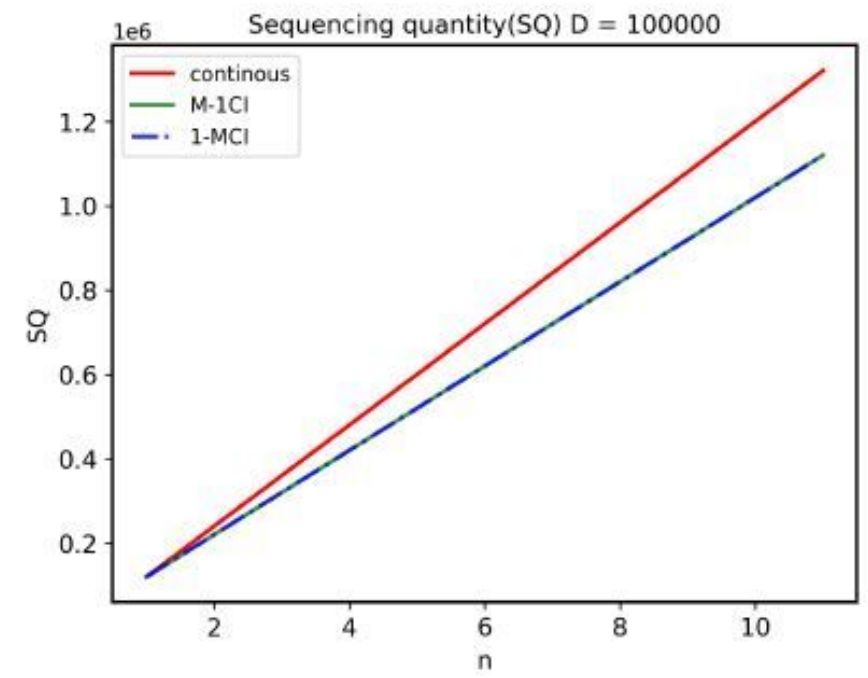

\section{Figure 8}

Comparison of data volume and sequencing volume We set the parameters as $\mathrm{Sh}=10 \mathrm{~B}, \mathrm{ST}=2010 \mathrm{~B}, \mathrm{Lp}$ $=20 \mathrm{bp}, \mathrm{Ls}=250 \mathrm{bp}$, and $\mathrm{a}=1=1: 7$ to obtain a comparison chart in the two cases of $\mathrm{D}=10 \mathrm{~KB}$ and $\mathrm{D}=$ $1 \mathrm{MB}$. Figures $8 \mathrm{a}$ and $8 \mathrm{bfollow}$ As $\mathrm{n}$ increases, the amount of binary data changes in the three methods. Figure $8 \mathrm{c}$ and Figure $8 \mathrm{~d}$ show the changes in the amount of sequencing of the three methods as $n$ increases. 